\title{
Numerical research of metal tungsten fluoride process
}

\author{
Roman Brendakov ${ }^{1}$, Alexander Shvab ${ }^{1}$, and Vladimir Brendakov ${ }^{1, *}$ \\ ${ }^{1}$ National Research Tomsk State University, 634030 Tomsk, Russia
}

\begin{abstract}
In this paper we consider the mathematical modelling of the process of obtaining tungsten hexafluoride gas, based on the reaction between tungsten powder and fluorine in a chemical reactor, which has a rectangle cross section channel as a working area. The modelling is conducted on the basis of solving three-dimensional equations of impulse, heat and substance transfer in the binary compound, taking into account the heterogeneous reaction undergoing on the bottom of the channel, between the tungsten powder layer and the fluorine gas flow. As a result of numerical solving the problem, the speed, concentration and temperature vector fields' distributions were revealed, as well as integral characteristic of the completeness of fluorine use. The credibility of the results obtained is confirmed by test researches and the comparing of calculation results with the empirical evidence.
\end{abstract}

\section{Introduction}

The obtaining of wear-resistant coatings, products made of refractory complex form materials or other complex parts of machines, working at high temperatures, pressures and shearing stresses is a pressing issue nowadays. As it is known, in practice there are lots of problems in the mechanical processing of refractory materials. However, the most promising way of getting wear-resistant coatings or products made of refractory materials has recently been a method, based on their precipitation from steam-gas phase with the help of the recovery of their fluorides or chlorides [1]. To carry out this method, it is necessary to initially undertake the process of fluoridation of metal tungsten. That is why the first phase of this method is the process of getting gaseous tungsten hexafluoride, which is formed as a result of the reaction between gaseous fluorine and tungsten powder. The process of tungsten fluoridation has the following reaction,

$$
W+3 F_{2}=W F_{6}
$$

which starts taking place at about $300{ }^{\circ} \mathrm{C}$.

In the work the numerical calculation of tungsten powder fluoridation in the chemical reactor was made, which has an extended rectangle cross section channel as a working area.

\footnotetext{
*Corresponding author: vnbrendakov@mephi.ru
} 


\section{Mathematical model of the process}

This process of tungsten powder fluoridation is made due to undergoing of the gaseous fluorine above the layer of tungsten powder, which is at the bottom of the channel. Going through the active zone of the reactor, fluorine interacts with tungsten powder, forming tungsten hexafluoride. The aim of the work was the research of hydrodynamics and thermal mass transfer influence on the process of getting gaseous metal tungsten hexafluoride. To reveal this influence, the numerical solving of three-dimensional equation system of Navier-Stokes of heat and substance transfer with a heterogeneous reaction on the lower surface of the reactors working area was made. For more full use of fluorine in a chemical reaction, a very low speed of its movement in the reactor is set. Therefore, the components of the binary compound can be considered as an incompressible environment. However, the density of the compound changes sufficiently due to the change of concentrations of the compound components due to the chemical reaction (1). That is why we consider the density a variable, though dependant only on the compound components concentrations and using the concept of additivity for gaseous compounds, we assume the compound density to be:

$$
\rho_{\mathrm{sm}}=\rho_{f} m_{f}+\rho_{\mathrm{g}} m_{\mathrm{g}}
$$

where $m_{f}$ and $m_{g}$ are voluminous concentrations of fluorine and tungsten hexafluoride accordingly. If we take fluorine density as a scale and consider for the binary compound that $m_{f}+m_{g}=1$, then we will have a dimensionless compound density pas a result, which is

$$
\rho=k_{\rho}+\left(1-k_{\rho}\right) m_{f}
$$

We impose relative mass fluorine concentrations $\varepsilon_{f}$ and tungsten hexafluoride $\varepsilon_{g}$. Expressing the compound density through the mass concentration $\varepsilon_{f}$ [2], we get:

$$
m_{f}=\frac{\varepsilon_{f}}{\varepsilon_{f}+\frac{M_{f}}{M_{g}}\left(1-\varepsilon_{f}\right)}
$$

where $M_{f}$ and $M_{g}$ are molecular masses of fluorine and tungsten hexafluoride accordingly.

One of the efficient ways of solving equations of environment movement in the derivatives "speed-pressure" is a method of physical fission along the time of speed and pressure fields.

\section{Numerical results}

The credibility of the numerical solving of the liquid dynamics for the set course in the square section channel was defined by the matching with the analytical solution, which is presented in the Fig. 1. The analytical solution has the following form [3]:

$$
u_{x}=\sum_{i=1,3,5 \ldots}^{\infty} \sum_{j=1,3,5 \ldots}^{\infty} \frac{\sin \left(\frac{\pi y i}{b}\right) \sin \left(\frac{\pi z j}{h}\right)}{i j\left(\frac{i^{2}}{b^{2}}+\frac{j^{2}}{h^{2}}\right)} .
$$


Here, $u_{x}$ is a ratio of dimensional speed to mid-dimensional and the dimensionless square sides are $b=B / H=1$ and $h=H / H=1$.

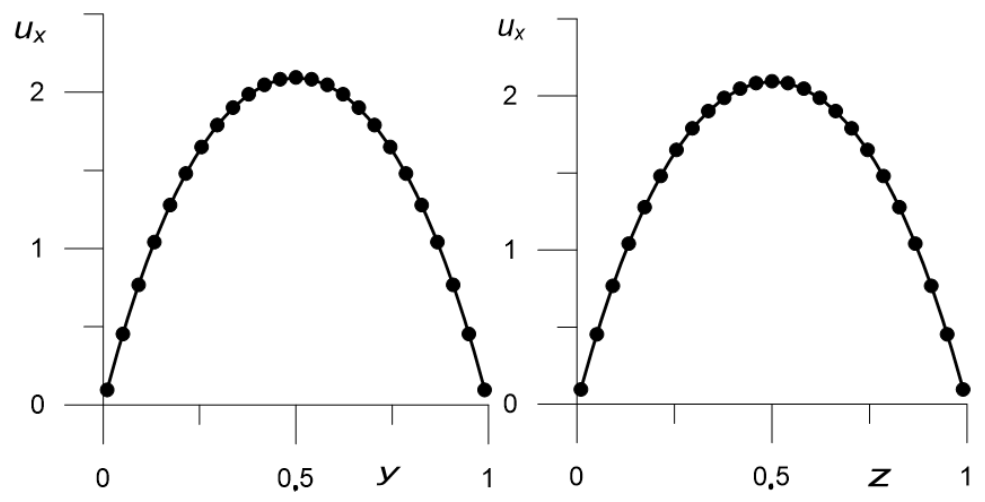

Fig.1. Comparison of the calculation (solid line) with the analytical dependence (2), shown by points, for steady flow in a channel with a square cross-section, a - on the central line in $\mathrm{z}, \mathrm{b}$ - on the center line along $\mathrm{y}$.

The credibility of the physics-mathematics model of the process of tungsten fluoridation offered was checked by comparing with experimental data [4] in distribution of the crosssection channel middle value of the mass concentration of tungsten hexafluoride, depending on the dimensionless channel length $x=X / L$. The comparison of the calculated and experimental data is shown in Fig.2.

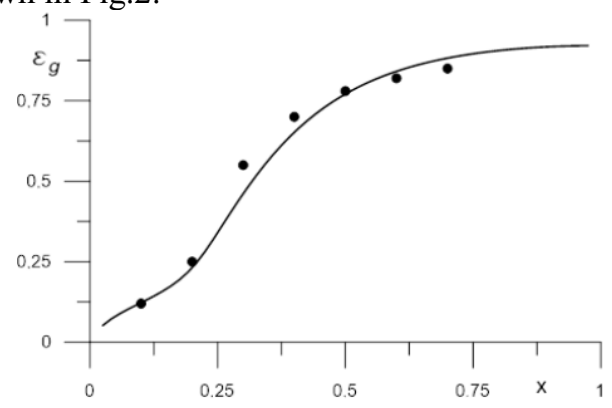

Fig. 2. The distribution of the average concentration of the concentration $\varepsilon_{\mathrm{g}}$ of tungsten hexafluoride as a function of the dimensionless length of the reaction zone $x$ for parameters $\operatorname{Re}=11 ; k_{\rho}=7.6 ; m=2$; $\operatorname{Pr}=1 ; \operatorname{Pr}_{d}=1 ; k_{\mu}=0.75 ; U=32951 \mathrm{~J} /$ mole $; k_{0}=438 ; k_{c}=0.45$.

The distribution of the isolines of the relative mass concentration $\varepsilon_{f}$, temperature isolines $\vartheta$ and compound densitypin the OXZ plane for the middle section of the channel, so $y=0.5$ is shown accordingly in Fig.3-Fig. 4 .
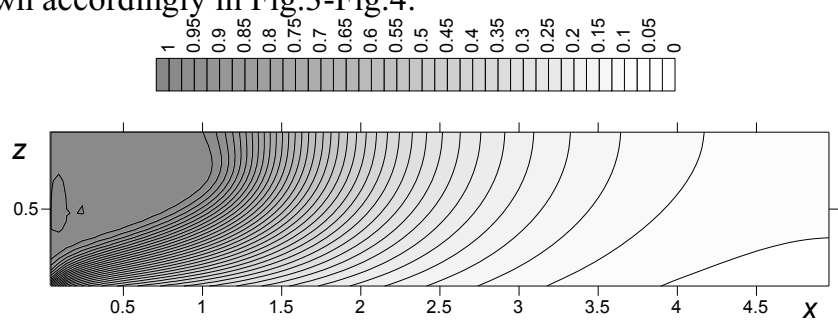

Fig. 3. The distribution of the isolines of the relative mass concentration of fluorine $\varepsilon_{f}$ in the middle section of the channel $y=0.5$. 


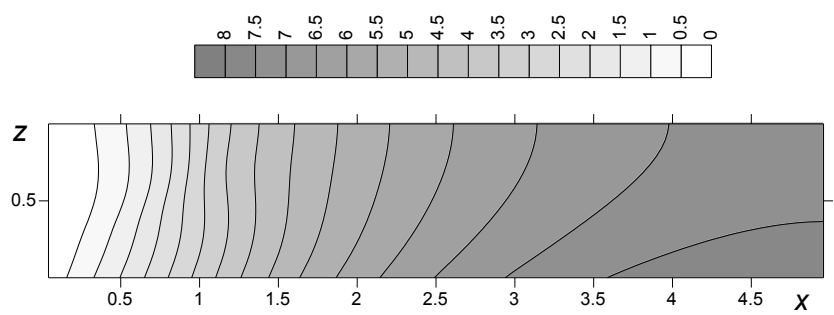

Fig. 4. The distribution of temperature isolines $\vartheta$ in the middle section of the channel $y=0.5$.

The adequacy of the model created is confirmed by the results, shown in the Fig.1-2. In the Fig. 3-4 the typical distribution of the mass fluorine and temperature concentration in the reactor is depicted. As it is seen from the figures, the process of stationary layer of tungsten powder fluoridation by breezing it with gas fluorine is quite mixed. This is primarily connected with the non-linear temperature field nature along the working area of the chemical reactor due to the additional thermal flow output, connected with endothermic reaction, as well as the significant increase of the compound density and as a result, blocking the access of the fluorine component to the reaction area, An important factor, influencing on the efficiency of fluoridation process is the formation of the diffusion border layer, which is the reason for the hexafluoride formation speed decrease. A complicated non-linear nature of the fluoridation process implementation doesn't allow to effectively apply methods of the planning of the experiments for getting regressive dependencies, connecting the main indicators of the process with regulated factors (the fluorine expense, the process temperature, etc.) As a conclusion, mathematical modeling is the only way to get reliable qualitative information about the process of tungsten powder fluoridation by the elementary fluorine [5].

\section{Conclusion}

The developed mathematical model can be used for the optimization of the fluoridation process in chemical reactors, as well as it can become a source of getting new detailed information about the mass, impulse and energy transferring processes, which opens the perspectives for creating new original technological decisions, meanwhile providing a higher level of environment protection [6].

\section{References}

1. J.-H. Park, J. Chem. Eng. 19, 391 (2002)

2. K. F. Pavlov, P. G. Romankov, A. A. Noskov, Examples and tasks in the course of processes and machines of chemical technology (Chemistry, Moscow, 1981) [in Russian]

3. B. S. Petuhov, Heat exchange and resistence in laminar course of liquid in tubes (Energy, Moscow, 1967) [in Russian]

4. Yu. M. Korolev, Russian journal of non-ferrous metal Eng. 4, 23 (2016)

5. V. N. Brendakov, A. V. Shvab, A. A. Demidenko, N. S. Evseev, R. V. Brendakov Patent No. 2641596 Way of getting tungsten product by applying tungsten layer by layer and device for its implementation (2016)

6. Yu. M. Korolev, Russian journal of non-ferrous metal Eng. 6, 29 (2016) 